\title{
Effect of Cytotoxicity of Pegylated Liposomal Recombinant Human Erythropoietin-alfa on Neuroblastoma Cell Line SH- SY5Y
}

\author{
Samaneh Shahbaz Hoseineh ${ }^{1}$, Azim Akbarzadeh ${ }^{2 *}$ and Hossein Attar ${ }^{1}$ \\ ${ }^{1}$ Department of Chemical Engineering, Science and Research Branch, Islamic Azad University, ${ }^{2}$ Department of Pilot \\ Nanobiotechnology, Pasteur Institute of Iran, Tehran, Iran \\ *For correspondence: Email: azimakbarzadeh1326@gmail.com; Tel: +989128387017, +982166465406; Fax: +982166465132
}

\begin{abstract}
Purpose: To evaluate the cytotoxic effect of pegylated liposomal Recombinant Human Erythropoietinalfa (rHuEPO) nanoparticles synthesized by reverse phase evaporation technique on SH-SY5Y cell line. Methods: To prepare the nanoparticles of the drug, rHuEPO, PEG3000, cholesterol and phosphatidylcholine were dissolved in buffer phosphate. The characteristics of the synthesized nanoparticles were determined by a zetasizer. Encapsulation efficiency, drug loading efficiency and drug release pattern were evaluated spectrophotometrically. The cytotoxicity effect of pegylated nanoliposomal rHuEPO was evaluated on SH-SY5Y cell line by 3-(4,5-dimethylthiazol-2-yl)-2,5diphenyltetrazolium bromide (MTT) assay.

Results: Size and zeta potential of pegylated nanoliposomal drug and blank pegylated nanoliposomes were $571 \pm 6.8 \mathrm{~nm},-16.5 \mathrm{mV} ; 280 \pm 4.71 \mathrm{~nm}$ and $-27.1 \mathrm{mV}$, respectively. Encapsulation and drug loading efficiency were $89 \pm 3.9 \%$ and $0.8 \pm 0.012 \%$, respectively. Drug release data indicate that 17 $\%$ of rHuEPO was released from pegylated liposomal nanoparticles over a period of $30 \mathrm{~h}$. The difference in cytotoxicity between the free drug $\left(I C_{50}=110.1 \pm 3.1 \mu \mathrm{g} / \mathrm{ml}\right)$ and nanodrug $\left(I C_{50}=87.2 \pm\right.$ $2.3 \mu \mathrm{g} / \mathrm{ml})$ was statistically significant $(p<0.05)$.

Conclusion: This study shows that pegylated nanoliposomal rHuEPO has a powerful cytotoxic effect on SH-SY5Y cell line and is therefore a suitable alternative to the standard therapy, rHuEPO, for the chemotherapy of neuroblastoma.
\end{abstract}

Keywords: Recombinant Human Erythropoietin-alfa, Cytotoxicity, Drug delivery, Liposome, Reverse phase evaporation, SH-SY5Y cell line

Tropical Journal of Pharmaceutical Research is indexed by Science Citation Index (SciSearch), Scopus, International Pharmaceutical Abstract, Chemical Abstracts, Embase, Index Copernicus, EBSCO, African Index Medicus, JournalSeek, Journal Citation Reports/Science Edition, Directory of Open Access Journals (DOAJ), African Journal Online, Bioline International, Open-J-Gate and Pharmacy Abstracts

\section{INTRODUCTION}

Recombinant human erythropoietin-alfa (rHuEPO) - a 165 amino acid glycoprotein is manufactured by recombinant DNA technology. rHuEPO is one of the most important drugs used in chemotherapy [1]. Additionally, non-targeted distribution of drug in living systems results in reducing efficiency and increasing side effects. Several side effects were reported to be associated with rHuEPO in consumers, such as skin rash or hives, edema (swelling of face, fingers, ankles, feet or lower legs), fever, hyperkalemia and increasing blood pressure. An effective way of overcoming the mentioned challenges is the encapsulation of drugs in nanocarriers, such as nanoliposomes.

Drug delivery is an effective approach of drug distribution to achieve therapeutic effect on 
humans. Liposomes which are capable of encapsulating a broad range of drugs can be used as drug carriers in such systems.

Liposomes are defined as vesicles that result from phospholipids dispersed in aqueous medium in such a way that the aqueous filled center cavity is surrounded by phospholipid bilayer membranes. The carriers are small vesicles of spherical shape and are also one of the nano drug carriers that improve the delivery of therapeutic agents which are loaded with great variety of molecules including drugs [2-4]. Liposomes are biodegradable, biocompatible, biologically inert and non-toxic vesicles, and they can encapsulate both hydrophilic and hydrophobic drugs. They are also nonantigenic and nonpyrogenic [5].

In the current study, we synthesized liposomal rHuEPO nanoparticles by reverse phase evaporation technique to increase efficiency and decrease side effect of the drug. We have also used polyethylene glycol (PEG) because of its effect on improving stability $[6,7]$.

\section{EXPERIMENTAL}

\section{Materials}

Phosphatidylcholine, cholesterol, polyethylene glycol 3000 (PEG3000) and MTT (0.5 mg/mL) were purchased from Sigma (USA). Isopropanol and ethanol were obtained from Merck Company (Germany). RPMI-1640 culture medium was purchased from Invitrogen (Invitrogen, USA). rHuEPO and neuroblastoma cell lines SH-SY5Y were supplied by Pasteur Institute of Iran. All other materials were analytical grade.

\section{Preparation of nanoparticles}

Nanoparticles were synthesized by reverse phase evaporation technique. rHuEPO, PEG3000, cholesterol and phosphatidylcholine (1:2.8:23:97 weight ratio) were dissolved in 50 $\mathrm{mL}$ of $98 \%$ ethanol using magnetic stirrer (150 rpm, $30 \mathrm{~min}$, room temperature) to obtain a transparent yellow suspension. Then, the solvent phase was evaporated by rotary evaporator (90 rpm, $1 \mathrm{~h}, 50{ }^{\circ} \mathrm{C}$ ) (Heidolph Co., Germany). Afterwards, the resultant film was dissolved in 10 $\mathrm{mL}$ buffer phosphate $(\mathrm{pH}=7.4)$. The control solution was also prepared without drug. The formulations were stirred for $24 \mathrm{~h}$ (150 rpm, room temperature) and sonicated (Bandelin Sonopuls HD 2070, Bandelin Elec., Germany) for $5 \mathrm{~min}$ at $60 \mathrm{~Hz}$. In addition, the counterpart concentrations of standard drug were prepared in phosphate buffer $(\mathrm{pH}=7.4)$.

\section{Characterization of nanoparticles}

The mean diameter, size distribution and Zeta potential of particles were determined by Zetasizer (Zen 3600; Malvern Instruments Ltd, Malvern Worcestershire, UK). Morphology of nanoparticles was studied by scanning electron microscope (AIS2300; Seron Technologies Inc.; Korea).

Encapsulation and drug loading efficiency were computed as in Eqs 1 and 2. For this purpose, 2 $\mathrm{mL}$ of each suspension were centrifuged (15000 rpm, $\left.4{ }^{\circ} \mathrm{C}, 90 \mathrm{~min}\right)$. Thereafter, the absorbance of the supernatant of both formulations was obtained spectrophotometrically (UV-Vis 1601PC, Shimadzu Co., Japan) at $280 \mathrm{~nm}$.

Encapsulation efficiency $(\%)=\left\{\left(C_{i}-C_{s}\right) / C_{i}\right\} 100 \ldots$

where $C_{i}$ is the initial concentration of the drug in $\mathrm{mg} / \mathrm{ml}$ and $C_{s}$ is the concentration of the drug in supernatant in $\mathrm{mg} / \mathrm{ml}$.

Loading efficiency $(\%)=\left(\mathrm{N}_{\mathrm{a}} / \mathrm{N}_{\mathrm{w}}\right) 100$

where $\mathrm{N}_{\mathrm{a}}$ is the amount of drug in the nanoparticle and $\mathrm{N}_{\mathrm{w}}$ is the weight of the nanoparticle all in $\mathrm{mg}$.

\section{In vitro drug release studies}

Drug release was determined by membrane diffusion technique. For this purpose, $1 \mathrm{~mL}$ of each formulation (pegylated nanoliposomal rHuEPO and the standard form) was poured in dialysis bag (cut off 50000 Dalton, Sigma). The dialysis bag was submerged in phosphate buffer $(\mathrm{pH}=7.4)$ and stirred $\left(100 \mathrm{rpm}, 37^{\circ} \mathrm{C}\right)$. At predetermined time intervals, $1 \mathrm{~mL}$ of phosphate buffer was taken and then substituted with fresh phosphate buffer. As a result, the amounts of released drug in phosphate buffer were measured using spectrophotometric method at definite time intervals.

\section{Evaluation of cytotoxicity}

The cytotoxicity of pegylated nanoliposomal rHuEPO on neuroblastoma cell line SH-SY5Y was investigated by MTT assay in 96-well plate reader. The cells were cultured using $1 \times 10^{4}$ cells in each well in RPMI-1640 containing $10 \%$ fetal bovine serum and $1 \%$ penicillin/streptomycin $\left(37^{\circ} \mathrm{C}, 5 \% \mathrm{CO}_{2}\right)$. Twenty four hours later -when cells adhered to the floor of the wells, cells were treated with standard 
rHuEPO and rHuEPO loaded nanoparticles in concentrations of $0.008,0.016,0.0323,0.0646$, $0.129,0.258,0.516,1.033$ and $2.066 \mu \mathrm{M}$. After incubating for $48 \mathrm{~h}$, supernatant was removed, and $100 \mu \mathrm{M}$ MTT solution $(0.5 \mathrm{mg} / \mathrm{mL}, \mathrm{pH}=7.4)$ was added to each well and incubated for $3 \mathrm{~h}$. Later, MTT solution was removed, and $200 \mu \mathrm{M}$ of isopropanol $100 \%(\mathrm{v} / \mathrm{v})$ was added to dissolve formazan crystals. Absorbance was determined at $570 \mathrm{~nm}$ (BioTek Instruments, VT, USA). Experiments were repeated three times. $I C_{50}$ was calculated by the statistical package, Pharm-PCS software.

\section{Statistical analysis}

SPSS version 21.00 software was used, and student t-test was applied to comparing statistical differences and $p<0.05$ was considered significant.

\section{RESULTS}

\section{Characteristics of nanoparticles}

The mean diameter, size distribution and zeta potential of blank pegylated liposome nanoparticles and pegylated liposomal rHuEPO are outlined in Table 1. As the values show, incorporation of drug into nanoparticles increased the size, size distribution and zeta potential of the nanoparticles.
The encapsulation and drug loading efficiency were $89 \pm 3.9$ and $0.8 \pm 0.012 \%$, respectively.

\section{In vitro drug release}

The drug release data for rHuEPO from pegylated nanoliposomal and standard drug are shown in Figure 2.

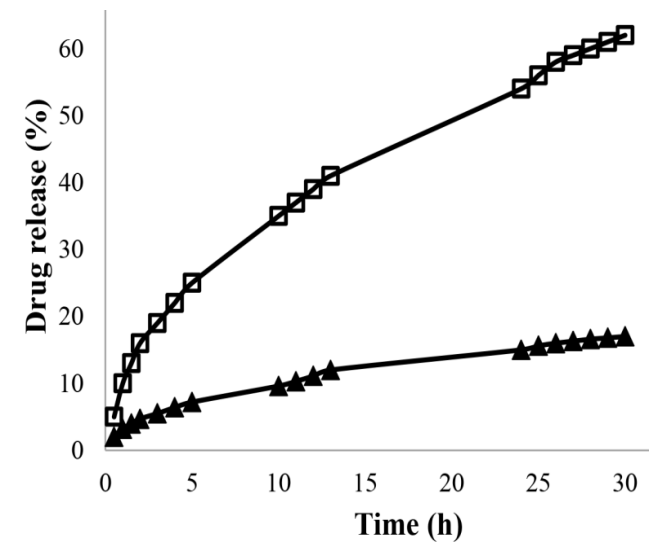

Figure 2: The release pattern of rHuEPO from pegylated liposomal drug nanoparticles synthesized by reverse phase evaporation technique $(\boldsymbol{\Delta})$ and standard drug ( $\square$ ). Pattern is presented as a percentage of release at different time intervals. Results are presented as mean $\pm 5 \%$ error from three independent tests

Table 1: Physicochemical properties of pegylated nanoliposomal rHuEPO

\begin{tabular}{lccc}
\hline Formulation & $\begin{array}{c}\text { Mean diameter } \\
(\mathbf{n m})^{*}\end{array}$ & $\begin{array}{c}\text { Zeta potential } \\
(\mathbf{m V})^{*}\end{array}$ & $\begin{array}{c}\text { Polydispersity } \\
\text { Index (PDI) }\end{array}$ \\
\hline $\begin{array}{l}\text { Pegylated liposomal } \\
\text { nanoparticles without drug }\end{array}$ & $280 \pm 4.71$ & -27.1 & 0.431 \\
$\begin{array}{l}\text { Pegylated liposomal } \\
\text { nanoparticles with drug }\end{array}$ & $571 \pm 6.8$ & -16.5 & 0.505 \\
\hline All data are presented as mean $\pm S D(n=3)$ & &
\end{tabular}

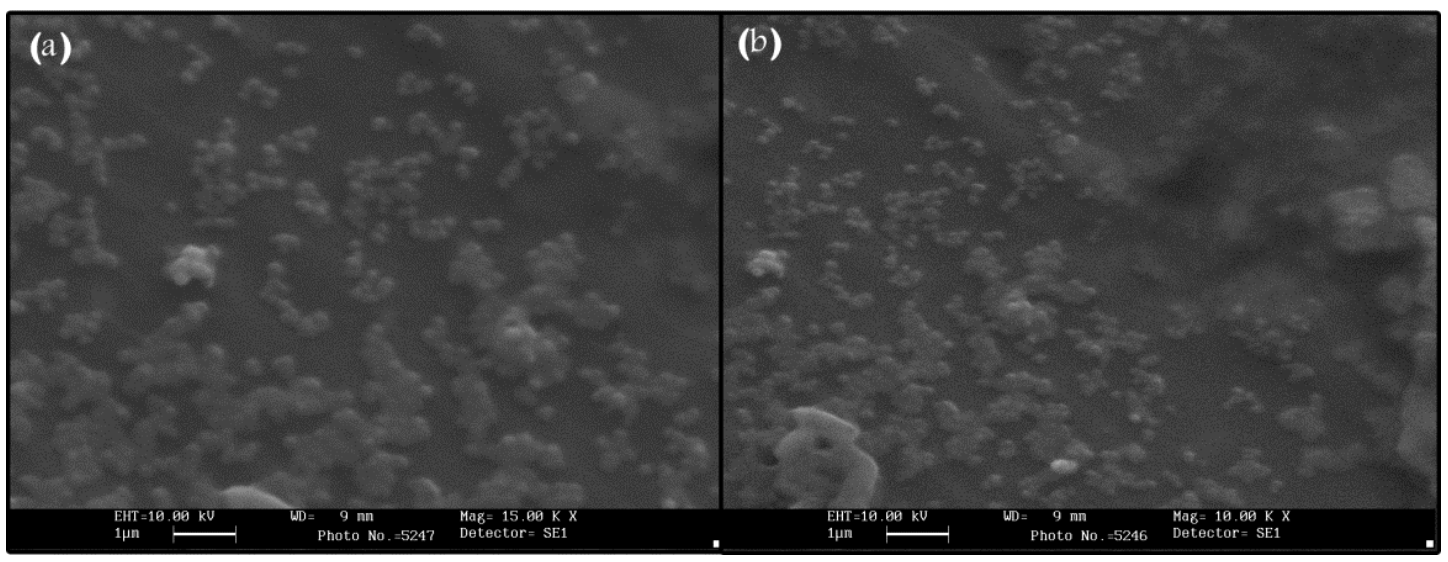

Figure 1: SEM image of pegylated liposomal rHuEPO nanoparticles (a) and blank pegylated liposomal nanoparticles (b) synthesized by reverse phase evaporation technique 


\section{Evaluation of cell cytotoxicity}

The $\mathrm{IC}_{50}$ for pegylated nanoliposomal rHuEPO and the standard form was $87.2 \pm 2.3 \mu \mathrm{g} / \mathrm{ml}$ and $110.1 \pm 3.1 \mu \mathrm{g} / \mathrm{ml}$, respectively.

\section{DISCUSSION}

Liposome drug delivery systems play an important role in the formulation of potent drugs to improve therapeutics. Currently, drug-loaded liposome nanoparticles are designed to reduce the side effects of therapeutic agents and also increase the drug accumulation in the target sites in some clinical applications [8]. In addition, liposomes could bypass the biological barriers whilst protecting entrapped drugs and deliver them to target organs, and it helps release of the drug at the therapeutic level [9].

Successful preparation of liposomal nanoparticles through reverse phase evaporation technique was achieved. The experiment was performed three times and results showed that the preparation technique was sufficiently valid and reliable.

Also, PEG was used in the synthesis of nanoparticles, because it is stable in the blood circulatory system and is not easily removed by the reticulo endothelial system (RES). In addition, PEG helps to increase pharmacokinetic properties and change insoluble drugs to soluble ones [10-13].

The results of the particles diameter measurement using Zetasizer after their fabrication confirmed the particles size in the nano scale [14]. Increasing the mean diameter of nanoparticles from 280 to $571 \mathrm{~nm}$ led to reduced stability due to the loaded drug in the nanoparticles.

The release process consisted of two phases, quick and slow release. In both formulations, a burst release of drug occurred in the first $3 \mathrm{~h}$. Release rate decreased with time which is probably because of presence of PEG. The results indicated $62 \pm 3.1 \%$ and $17 \pm 0.72 \%$ of the drug were released into phosphate buffer for the standard drug and pegylated liposomal drug, respectively. Previous studies showed PEG also brings about increased stability and probability of drug delivery to tumor with high efficiency [15].

The cytotoxicity of pegylated liposomal rHuEPO nanoparticles on neuroblastoma cell line $\mathrm{SH}$ SY5Y was evaluated by the MTT assay. Firstly, it was determined that nanoparticles containing no drug had no cytotoxic effect on the cells. The results showed that pegylated liposomal formulation had more toxic effect compared with the standard drug. Regarding definition of $\mathrm{IC}_{50}$, the cytotoxicity of the new formulation was estimated to be 1.3 times more than the standard drug for SH-SY5Y cell line.

\section{CONCLUSION}

Nanotechnology-based drug delivery systems, especially liposomes, are currently a rapidly advancing field of science. Pegylated nanoliposome particles have been successfully synthesized by reverse phase evaporation technique in this study. Significant improvement in the efficiency of pegylated liposome nanoparticles rHuEPO compared with the standard form of the drug has been achieved. Further studies on the formulation including in vivo experiments are recommended.

\section{REFERENCES}

1. Auerbach M, Ballard H, Trout JR, Mcllwain M, Ackerman A, Bahrain H, Balan S, Barker L, Rana J. Intravenous iron optimizes the response to recombinant human erythropoietin in cancer patients with chemotherapyrelated anemia: a multicenter, open-label, randomized trial. J Clin Oncol 2004; 22: 1301-1307.

2. Samad A, Sultana Y, Aqil M. Liposomal drug delivery systems: an update review. Curr Drug Deliv 2007; 4: 297-305.

3. Allen TM, Cullis PR. Liposomal drug delivery systems: from concept to clinical applications. Adv Drug Deliv Rev 2013; 65: 36-48.

4. Bangham $A D$, Standish MM, Watkins JC. Diffusion of univalent ions across the lamellae of swollen phospholipids. J Mol Biol 1965; 13: 238-252.

5. Dhandapani NV, Thapa A, Sandip G, Shrestha A, Shrestha N, Bhattarai RS. Liposomes as novel drug delivery system: A comprehensive review. Int $J$ Res Pharm Sci 2013; 4: 187-193.

6. Ryan SM, Mantovani G, Wang $X$, Haddleton DM, Brayden DJ. Advances in PEGylation of important biotech molecules: delivery aspects. Expert Opin Drug Deliv 2008; 5: 371-383.

7. Nakase I, Lai $H$, Singh NP, Sasaki T. Anticancer Properties of Artemisinin Derivatives and Their Targeted Delivery by Transferrin Conjugation. Int $J$ Pharm 2008; 354: 28-33.

8. Lian $T$, Ho RJ. Trends and developments in liposome drug delivery systems. J Pharm Sci 2001; 90: 667680.

9. Costantino $L$, Boraschi D. Is there a clinical future for polymeric nanoparticles as brain-targeting drug delivery agents? Drug Discov Today 2012; 17: 367378. 
10. Cervantes F, Mesa R, Barosi G. New and old treatment modalities in primary myelofibrosis. Cancer $J$ 2007; 13: $377-383$

11. Kawai F. Microbial degradation of polyethers. Appl Microbiol Biotechnol 2002; 58: 30-38.

12. Veronese FM, Pasut G. PEGylation, successful approach to drug delivery. Drug Discov Today 2005; 10: 14511458.

13. Matteucci ML, Anyarambhatla G, Rosner G, Azuma C, Fisher PE, Dewhirst MW, Needham D, Thrall DE.
Hyperthermia increases accumulation of technetium$99 m$-labeled liposomes in feline sarcomas. Clin Cancer Res 2000; 6: 3748-3755.

14. Mansour HM, Rhee YS, Wu X. Nanomedicine in pulmonary delivery. Int J Nanomedicine 2009; 4: 299319.

15. Otsuka H, Nagasaki Y, Kataoka K. PEGylated nanoparticles for biological and pharmaceutical applications. Adv Drug Deliv Rev 2003; 55: 403-419. 